\title{
Loop-free construction of counter-models for intuitionistic propositional logic
}

\author{
Luis Pinto and Roy Dyckhoff*
}

\begin{abstract}
We present a non-looping method to construct Kripke trees refuting the nontheorems of intuitionistic propositional logic, using a contraction-free sequent calculus.
\end{abstract}

1991 Mathematics Subject Classification: 03B20, 03B35, 03C25, 03F03, 68T15

\section{Introduction}

It is well known that IPL (Intuitionistic Propositional Logic) has the finite model property; in fact, any non-theorem of IPL can be invalidated by means of a finite Kripke tree [2]. The standard method (see [9] for a formal treatment) for constructing such counter-models requires a loop-checker. Here, we present a method for constructing counter-models not requiring a loop-checker, based on the contractionfree sequent calculi LJT and L.JT* ${ }^{*}[1]$.

LJT provides a very simple but reasonably effective decision procedure for IPL. The ideas were first presented in the work of Vorob'ev [10] and more recently also in [3] and [5]. LJT differs from traditional formulations of Gentzen sequent calculi for IPL only in the rule $\supset \Rightarrow$ for introduction of implication on the left and in not having a contraction rule as primitive. Traditionally, during the proof-search, in going from the conclusion of $\supset \Rightarrow$ to its major premiss, we have to duplicate the principal formula, raising the problem of non-termination. In LJT the rule $\supset \Rightarrow$ is replaced by four other rules; the calculus is then terminating, in the sense that every increasing sequence of partial proofs terminates.

LJT $^{*}$ is a Beth-style (multi-succedent) variant of LJT, also shown in [1] to have the same termination property. It is not immediately obvious that it can be used effectively to build counter-models; our purpose in this paper is to show that it can. For expository reasons, we use LJT* to define a calculus CRIP (Calculus for Refutation of Intuitionistic Propositions) that captures unprovability for IPL. We show that each sequent is either a theorem of LJT* or a 'theorem' of CRIP. We show that CRIP is also terminating and we show how, for each 'theorem' of CRIP, to construct a finite Kripke tree witnessing the 'theoremhood'.

* Both authors acknowledge the support of ESPRIT grant BRA 7232 "GENTZEN"; the first author is supported by a grant from the JNICT, Portugal. 


\section{The calculus LJT*}

Atoms are denoted $p, q, \ldots$. Propositional formulae $A, B, \ldots$ are built up as usual using symbols for conjunction, disjunction and implication. We denote absurdity by $f$ and use it to define negation by $\neg A \stackrel{\text { def }}{=} A \supset f$. Sequents have the form $\Gamma \Rightarrow \Delta$, where the antecedent $\Gamma$ and the succedent $\Delta$ are multisets of formulae. L.JT* has no (primitive) structural rules. The logical rules are standard, with the exception of the rule $\supset \Rightarrow$ for introduction of implication on the left. This rule is divided into four cases, as in figure 1. (The exceptional rules for LJT are those obtained from those in figure 1 by allowing just a single formula in the succedent.) Notice that the left premiss of $\supset \Rightarrow_{4}^{*}$ contains only one formula in the succedent. All the rules in $\mathbf{L J T}^{*}$ apart from $\Rightarrow \supset^{*}$ (introduction of implication on the right) and $\supset \Rightarrow_{4}^{*}$ are invertible. LJT* formalises intuitionistic provability for sequents; [1] gives details of its correctness and completeness.

$$
\begin{gathered}
\frac{\Gamma, p, B \Rightarrow \Delta}{\Gamma, p, p \supset B \Rightarrow \Delta} \supset \Rightarrow_{1}^{*} \quad \frac{\Gamma, C \supset B, D \supset B \Rightarrow \Delta}{\Gamma,(C \vee D) \supset B \Rightarrow \Delta} \supset_{2}^{*} \\
\frac{\Gamma, C \supset(D \supset B) \Rightarrow \Delta}{\Gamma,(C \wedge D) \supset B \Rightarrow \Delta} \supset \Rightarrow_{3}^{*} \quad \frac{D \supset B, C, \Gamma \Rightarrow D \quad B, \Gamma \Rightarrow \Delta}{(C \supset D) \supset B, \Gamma \Rightarrow \Delta} \supset_{4}^{*}
\end{gathered}
$$

Figure 1: The LJT $^{*}$ rules for introduction of implication on the left.

Definition 1. An implication $A \supset B$ is an atomic implication, with atom $A$ if $A$ is atomic; it is a nested implication if $A$ has the form $C \supset D$.

Definition 2. A sequent $\Gamma \Rightarrow \Delta$ is irreducible if: (i) its antecedent $\Gamma$ contains only atomic implications, nested implications and atomic formulae and none of the atoms of the atomic implications are equal to any of the atomic formulae; (ii) its succedent $\Delta$ contains only implications and atomic formulae. $\Gamma \Rightarrow \Delta$ is strongly irreducible if it is irreducible and contains neither nested implications in the antecedent $\Gamma$ nor implications in the succedent $\Delta$.

Lemma 1. A strongly irreducible sequent $\Gamma \Rightarrow \Delta$ is provable in L.JT* iff it is an axiom of LJT* (i.e., $\Gamma$ and $\Delta$ have a formula in common).

Proof. Trivial.

\section{The calculus CRIP}

An anti-sequent is a pair $(\Gamma, \Delta)$ of multisets of formulae, represented as $\Gamma \nRightarrow \Delta$. Figure 2 presents the calculus CRIP formalising refutability of sequents of IPL 
as 'provability' (of the corresponding anti-sequent) in CRIP. We call the formal structures built up according to the rules of CRIP refutations.

$$
\begin{gathered}
\frac{p_{1} \supset B_{1}, \ldots, p_{n} \supset B_{n}, \Gamma \nRightarrow \Delta}{\text { axiom }^{*}} \\
\frac{\Gamma, A, B \nRightarrow \Delta}{\Gamma, A \wedge B \nRightarrow \Delta}(1) \frac{\Gamma \nRightarrow A, \Delta}{\Gamma \nRightarrow A \wedge B, \Delta}(2) \frac{\Gamma \nRightarrow B, \Delta}{\Gamma \nRightarrow A \wedge B, \Delta}(3) \\
\frac{\Gamma, A \nRightarrow \Delta}{\Gamma, A \vee B \nRightarrow \Delta}(4) \frac{\Gamma, B \nRightarrow \Delta}{\Gamma, A \vee B \nRightarrow \Delta}(5) \frac{\Gamma \not A, B, \Delta}{\Gamma \nRightarrow A \vee B, \Delta}(6) \\
\frac{\Gamma, p, B \nRightarrow \Delta}{\Gamma, p, p \supset B \nRightarrow \Delta}(7) \frac{\Gamma, C \supset B, D \supset B \nRightarrow \Delta}{\Gamma,(C \vee D) \supset B \nRightarrow \Delta}(8) \frac{\Gamma, C \supset(D \supset B) \nRightarrow \Delta}{\Gamma,(C \wedge D) \supset B \nRightarrow \Delta}(9) \\
\frac{B, \Gamma \nRightarrow \Delta}{(C \supset D) \supset B, \Gamma \nRightarrow \Delta}(10) \\
\frac{D_{1} \supset B_{1}, C_{1}, \Gamma_{1} \nRightarrow D_{1} \ldots D_{n} \supset B_{n}, C_{n}, \Gamma_{n} \nRightarrow D_{n} \Gamma^{\prime}, E_{1} \nRightarrow F_{1} \ldots \Gamma^{\prime}, E_{m} \nRightarrow F_{m}}{\Gamma^{\prime} \nRightarrow E_{1} \supset F_{1}, \ldots, E_{m} \supset F_{m}, \Delta}(11)^{\diamond}
\end{gathered}
$$

where in rule $(11) \Gamma^{\prime}=\left(C_{1} \supset D_{1}\right) \supset B_{1}, \ldots,\left(C_{n} \supset D_{n}\right) \supset B_{n}, \Gamma$ and $\Gamma_{i}=$ $\Gamma^{\prime} \backslash\left\{\left(C_{i} \supset D_{i}\right) \supset B_{i}\right\}$

The provisos in rules axiom and (11) are:

* $\Gamma$ and $\Delta$ contain only atomic formulae and are disjoint. Every $p_{i}$ is atomic and not in $\Gamma$. $\Gamma$ does not contain $f$.

$\diamond$ Every formula in $\Gamma$ is either atomic or an atomic implication and none of the antecedents of the atomic implications are equal to any of the atomic formulae. $\Delta$ contains only atomic formulae. $\Gamma$ and $\Delta$ are disjoint.

Figure 2: The calculus CRIP.

Lemma 2. The well-founded ordering on (anti-) sequents given in [1] is such that for each rule of CRIP each of the premises is smaller than the conclusion.

Proof. By inspection, as in [1]. Note that the ordering is just the Dershowitz-Manna multiset ordering induced by an obvious well-founded ordering on formulae.

Corollary 1. CRIP is terminating.

Proof. Trivial.

Theorem 1. For every pair $(\Gamma, \Delta)$ of multisets of formulae, either $\vdash_{L J T *} \Gamma \Rightarrow \Delta$ or $\vdash_{C R I P} \Gamma \nRightarrow \Delta$, but not both. 
Proof. Consider two multisets $\Gamma, \Delta$ of formulae. We show by induction (using the multiset ordering) on the multiset union $\Gamma \cup \Delta$ that either $\vdash_{L J T *} \Gamma \Rightarrow \Delta$ or $\vdash_{C R I P} \Gamma \nRightarrow \Delta$. There are several cases:

1. If $\Gamma \Rightarrow \Delta$ is strongly irreducible then either it is an axiom of $\mathbf{L} \mathbf{J} \mathbf{T}^{*}$, by virtue of an atom occurring in $\Gamma \cap \Delta$, or it is an axiom of CRIP: in each case, the conclusion follows.

2. Suppose $\Delta=A \wedge B, \Delta^{\prime}$. By the induction hypothesis, one of four cases holds: a) $\vdash_{L J T *} \Gamma \Rightarrow A, \Delta^{\prime}$ and $\vdash_{L J T *} \Gamma \Rightarrow B, \Delta^{\prime}$ and then, applying the rule for introduction of conjunction on the right, we get $\vdash_{L J T *} \Gamma \Rightarrow A \wedge B, \Delta^{\prime}$.

b) $\vdash_{L J T *} \Gamma \Rightarrow A, \Delta^{\prime}$ and $\vdash_{C R I P} \Gamma \nRightarrow B, \Delta^{\prime}$ and then by applying rule 3 we obtain $\vdash_{C R I P} \Gamma \nRightarrow A \wedge B, \Delta^{\prime}$.

c) $\vdash_{C R I P} \Gamma \nRightarrow A, \Delta^{\prime}$ and $\vdash_{L J T *} \Gamma \Rightarrow B, \Delta^{\prime}$ and then by applying rule 2 we obtain $\vdash_{C R I P} \Gamma \nRightarrow A \wedge B, \Delta^{\prime}$.

d) $\vdash_{C R I P} \Gamma \nRightarrow A, \Delta^{\prime}$ and $\vdash_{C R I P} \Gamma \nRightarrow B, \Delta^{\prime}$; we can then use either rule 2 or rule 3 to obtain $\vdash_{C R I P} \Gamma \nRightarrow A \wedge B, \Delta^{\prime}$.

3. The cases dealing with disjunctions in $\Delta$ or with conjunctions, disjunctions or implications whose antecedents are either conjunctions, disjunctions or atomic formulae in $\Gamma$ all follow by the same kind of argument; they all correspond to invertible rules in $\mathbf{L} \mathbf{J T} *$.

4. For the remaining case we have:

$$
\begin{gathered}
\Gamma=\left(C_{1} \supset D_{1}\right) \supset B_{1}, \ldots,\left(C_{n} \supset D_{n}\right) \supset B_{n}, \Gamma^{\prime} \\
\Delta=E_{1} \supset F_{1}, \ldots, E_{m} \supset F_{m}, \Delta^{\prime}
\end{gathered}
$$

where $\Delta^{\prime}$ contains only atomic formulae and $\Gamma^{\prime}$ contains only atomic formulae and atomic implications. $\Gamma_{i}$ will stand for $\Gamma-\left(C_{i} \supset D_{i}\right) \supset B_{i}$. If, for some $1 \leq i \leq m, \vdash_{L J T *} \Gamma, E_{i} \Rightarrow F_{i}$, then using the rule $\Rightarrow \supset^{*}$ for introduction of implication on the right we obtain $\vdash_{L J T *} \Gamma \Rightarrow \Delta$. If, for some $1 \leq i \leq n$, $\vdash_{C R I P} \Gamma_{i}, B_{i} \nRightarrow \Delta$, then applying rule 10 we obtain $\vdash_{C R I P} \Gamma \nRightarrow \Delta$. Otherwise, by induction, for all $1 \leq i \leq m \vdash_{C R I P} \Gamma, E_{i} \not F_{i}$ and for all $1 \leq i \leq n$ $\vdash_{L J T *} \Gamma_{i}, B_{i} \Rightarrow \Delta$. If, for some $1 \leq i \leq n$ we have $\vdash_{L J T *} \Gamma_{i}, D_{i} \supset B_{i}, \bar{C}_{i} \Rightarrow D_{i}$, then using $\supset \Rightarrow_{4}^{*}$ we have $\vdash_{L J T *} \Gamma \Rightarrow \bar{\Delta}$; and if not, applying rule 11 we obtain $\vdash_{C R I P} \Gamma \nRightarrow \Delta$.

Thus, we have shown that for any pair $(\Gamma, \Delta)$ either $\vdash_{L J T *} \Gamma \Rightarrow \Delta$ or $\vdash_{C R I P} \Gamma \nRightarrow \Delta$. Also, it is routine to show by induction (on the size of the LJT* proof) that only one of these two can hold.

\section{Construction of counter-models}

We assume familiarity with Kripke semantics [4, 2, 7]. In brief, we are interested in finite Kripke trees, i.e., sets $K$ with a reflexive transitive binary relation $\leq$ on $K$, with a least element $w_{0}$ such that $K \backslash w_{0}$ is a disjoint union of Kripke trees, together with a monotone relation $\models$ from $K$ to the set of atoms of IPL, extendable in 
a standard way to a 'forcing' relation $\models$ between $K$ and the formulae of IPL. Elements of $K$ are called its worlds; $w_{0}$ is called the initial world.

Definition 3. A Kripke tree is a counter-model to a sequent $\Gamma \Rightarrow \Delta$ if it has a world in which all the formulae in $\Gamma$ are forced and none of the formulae in $\Delta$ are forced.

Definition 4. A Kripke tree is a strong counter-model to a sequent $\Gamma \Rightarrow \Delta$ if in its initial world all the formulae in $\Gamma$ are forced and none of the formulae in $\Delta$ are forced.

Every strong counter-model is a counter-model but not conversely. The method described below for constructing counter-models for the non-theorems of IPL in fact constructs strong counter-models.

The construction of counter-models is closely related to the notion of invertibility. For an invertible rule the conclusion is provable iff the premises are provable, for, a counter-model to the conclusion is the same as a counter-model to one of the premises. Non-invertible rules introduce choice points in the proof-search; this corresponds to branching in the counter-model. The base case is where none of the rules can be applied. In this case we proceed classically, forcing everything in the antecedent and none of the things in the succedent. We formalize this construction in lemma 4 and proposition 2.

For expository reasons, in what follows, we will be talking about counter-models (strong counter-models) to anti-sequents. A counter-model (strong counter-model) to a sequent $\Gamma \Rightarrow \Delta$ is the same as the counter-model (strong counter-model) our method constructs to $\Gamma \nRightarrow \Delta$.

Lemma 3. For any world $w$ in a Kripke tree $K$, if $A \supset B$ is a theorem of IPL and if $w \models A$ then $w \mid=B$.

Proof. Follows from definition.

Corollary 1. For any world $w$ in a Kripke tree, if $A$ and $B$ are provably equivalent formulae of IPL then $w \models A$ iff $w \models B$.

Proof. Trivial.

Lemma 4. Let $p_{1} \supset B_{1}, \ldots, p_{n} \supset B_{n}, \Gamma \not \Delta$, where $\Gamma$ contains only atoms, be an axiom of CRIP. Then the tree $K$ consisting only of the single world $w$ forcing the atoms in $\Gamma$ is a strong counter-model to the axiom.

Proof. Trivial.

Proposition 1. For every rule of CRIP, if there is a strong counter-model for each of the premises then there is a strong counter-model for its conclusion.

Proof. For the rules $1,2, \ldots, 10$, a strong counter-model to the conclusion of the rule is the same as a strong counter-model to its premiss(es). We prove that this holds for rule 9 ; all the other cases are easily proved in the same way. Let us assume 
that $K$, with initial world $w$, is a strong counter-model to $\Gamma, C \supset(D \supset B) \nRightarrow \Delta$. We have to prove that $K$ is also a strong counter-model to $\Gamma,(C \wedge D) \supset B \neq \Delta$. To do so it is enough to prove that $w \models(C \wedge D) \supset B$; this follows by corollary 1 because $C \supset(D \supset B)$ is equivalent to $(C \wedge D) \supset B$.

For rule 11 , suppose we have strong counter-models $K_{1}, \ldots, K_{n}, K_{1}^{\prime}, \ldots, K_{m}^{\prime}$ to the $n+m$ premises. A strong counter-model $K$ to the conclusion is a Kripke tree with initial world $w$ forcing all the atoms in $\Gamma$ and with $n+m$ children given by $K_{1}, \ldots, K_{n}, K_{1}^{\prime}, \ldots, K_{m}^{\prime}$. It is easy to prove that this construction yields a Kripke tree [7]. To show that $K$ is a strong counter-model we have to prove that $w$ forces all of the formulae in the antecedent and none of the formulae in the succedent. Recall that $\Gamma$ contains only atomic formulae, which are forced in $w$ by construction, and atomic implications, which are forced in $w$ because none of their antecedents can be forced, otherwise the anti-sequent would have been reducible. By hypothesis all the initial worlds of the Kripke trees $K_{1}, \ldots, K_{n}, K_{1}^{\prime}, \ldots, K_{m}^{\prime}$ force these atomic implications. Now we prove that for all $i: 1 \leq i \leq m w \models\left(C_{i} \supset D_{i}\right) \supset B_{i}$. We know that $w \forall C_{i} \supset D_{i}$ because, in the initial world of $K_{i}, C_{i}$ is forced and $D_{i}$ is not forced. We still have to prove $\left(C_{i} \supset D_{i}\right) \supset B_{i}$ is forced at each of the $K_{j}(1 \leq j \leq n+m)$. By hypothesis $\left(C_{i} \supset D_{i}\right) \supset B_{i}$ is certainly forced in all the $K_{j}$ except $K_{i}$. Because $C \wedge(D \supset B)$ implies $(C \supset D) \supset B$ applying lemma 3 we easily prove that $K_{i}$ also forces $\left(C_{i} \supset D_{i}\right) \supset B_{i}$. We still have to prove that none of the formulae in the succedent are forced in $w$. The atomic formulae cannot be forced since they are all distinct from the atomic formulae forced in $w$. None of the formulae $E_{i} \supset F_{i}$ can be forced in $w$ because, in the initial world of $K_{i}^{\prime}$, $E_{i}$ is forced but $F_{i}$ is not forced.

Example 1. We apply the method described above to construct a strong countermodel to $((p \supset q) \supset p) \supset p$ (Peirce's formula). Figure 3 presents a refutation in CRIP of the antisequent for Peirce's formula.

$$
\frac{\frac{q \supset p, p \not q}{(p \supset q) \supset p \not p}}{\not((p \supset q) \supset p) \supset p}
$$

Figure 3: CRIP refutation of Peirce's formula.

Applying proposition 1 and the observation that $\vdash_{L J T *} \Gamma \Rightarrow A \supset B$ iff $\vdash_{L J T *}$ $\Gamma, A \Rightarrow B$, (i.e., for this particular case the rule $\Rightarrow \supset^{*}$ for introduction of implication on the right is invertible, and so a counter-model to the conclusion is the same as a counter-model to the premiss), we see that the following Kripke tree witnesses the refutability of Peirce's formula. The tree has two worlds, $w_{1}$ and $w_{2}$, with $w_{1} \leq w_{2}$. In $w_{1}$ nothing is forced and in $w_{2} p$ is forced. It is easy to check that $w_{1}$ does not force $((p \supset q) \supset p) \supset p$.

In figure 4 we illustrate how Peirce's formula could be refuted using a method with loop-checking. The Kripke tree we obtain using this method is the same as the one obtained before. 


$\frac{\frac{\text { looping }}{(p \supset q) \supset p \not p \supset q} \frac{p, p \not q q}{(p \supset q) \supset p, p \nRightarrow q}}{(p \supset q) \supset p \nRightarrow p \supset q, p}$

Figure 4: Refutation of Peirce's formula with looping.

\section{Related work}

Tiomkin [7] gives a method to construct counter-models for the non-theorems in classical logic. Unlike in IPL, all the rules for classical propositional logic are invertible: this trivialises the construction of counter-models.

Underwood [9] presents a constructive proof of completeness of finite Kripke trees for IPL; the proof is formalized in Nuprl, which allows an algorithm to be extracted mechanically from the proof. This algorithm produces for each formula either a proof, in the form of an inhabitant of the corresponding type, or a finite Kripke tree and a world therein such that the formula is not forced in that world. The termination of this algorithm is proved by induction on the number of formulae and sequents that can be added to the system without generating repetitions; effectively, the implementation depends on a loop-checker.

Miglioli et al [6] exploit the calculi presented in [1] to give an improved proof procedure for intuitionistic predicate logic; although described as a refutation system, there is no explicit refutation calculus in the sense given by CRIP, and the exposition of the method of building Kripke interpretations is complicated by the need to handle first order formulae. (There are interesting applications to Kuroda logic.)

Remark 1. We regard Kripke trees as having the same relation to refutation trees in CRIP as lambda-terms have to sequent calculus or natural deduction proofs: they contain information from which one can readily observe the existence of a refutation (resp., proof) only by some fairly non-trivial steps.

\section{Conclusion}

In principle, everyone knows how to build Kripke trees, but in practice the technique involves loop-checking. We have shown how to build Kripke trees witnessing the non-provability (in IPL) of propositional formulae by a method which involves no loop-checking. 


\section{References}

[1] Dyckhoff, R., Contraction-free sequent calculi for intuitionistic logic. Journal of Symbolic Logic 57 (1992), 795-807.

[2] Fitting, M., Intuitionistic Logic, Model Theory and Forcing, North-Holland, Amsterdam, 1969.

[3] Hudelmaier, J., Bounds for cut-elimination in intuitionistic propositional logic. Archive for Mathematical Logic 31 (1992), 331-353.

[4] Kripke, S., Semantical analysis of intuitionistic logic I, In Formal systems and recursive functions, edited by J.N. Crossley and M.A.E. Dummett, pp 92-130, NorthHolland, Amsterdam, 1965.

[5] Lincoln, P., Scedrov, A. and Shankar, N., Linearising intuitionistic implication. In Sixth Annual IEEE Symposium on Logic in Computer Science, Amsterdam, Netherlands, pp 51-62, IEEE Computer Society Press, 1991.

[6] Miglioli, P., Moscato, P. and Ornaghi, O., How to avoid duplications in refutation systems for intuitionistic logic and Kuroda logic, Internal Report 99-93, Dip. di Scienze dell' Informazione, University of Milan, 1993.

[7] Smorynski, C.: Applications of Kripke models, In Metamathematical investigations of intuitionistic arithmetic and analysis, edited by A.S. Troelstra, Chapter V, Lecture Notes in Mathematics 344, Springer-Verlag, 1973.

[8] Tiomkin, M., Proving unprovability. In Third Annual IEEE Symposium on Logic in Computer Science, Edinburgh, Scotland, pp 22-26, IEEE Computer Society Press, 1988.

[9] Underwood, J., A constructive completeness proof for the intuitionistic propositional calculus. Technical Report 90-1179, Cornell University, 1990. Also in Proceedings of the Second Workshop on Theorem Proving with Analytic Tableaux and Related Methods, April 1993, Marseille, France.

[10] Vorob'ev, N.N., The derivability problem in the constructive propositional calculus with strong negation. Doklady Akademii Nauk SSSR, 85 (1952), 689-692. (In Russian). 